\title{
PROPUESTA METODOLÓGICA PARA CALENDARIZAR EL RIEGO EN INVERNADERO RUSTICO USANDO UN ÍNDICE DE ESTRÉS TÉRMICO
}

\section{A METHODOLOGICAL APPROACH FOR IRRIGATION SCHEDULING IN A RUSTIC GREENHOUSE BY USING A STRESS THERMAL INDEX}

\author{
José Alberto Urrieta-Velázquez ${ }^{1}$, Araceli Gómez-Zaldivar', \\ Angélica Gómez-Zaldivar ${ }^{1}$ y Jorge Flores-Velázquez ${ }^{2 *}$
}

\begin{abstract}
'Instituto Naconal de Investigaciones Forestales, Agrícolas y Pecuarias, CENID-RASPA, Ejido Las Huertas, Gómez Palacio, Durango, México. ${ }^{2}$ Colegio de Postgraduados, Campus Motecillo, Posgrado en Hidrociencias, Montecillo, Texcoco, Estado de México, México.

*Autor de correspondencia (jorgelv@colpos.mx)
\end{abstract}

\section{RESUMEN}

El uso de sensores y actuadores permite la aplicación precisa del riego a través de sistemas automatizados y retroalimentados, aumentando la eficiencia en el uso del agua; no obstante, su alto costo dificulta su uso en invernaderos de mediana a baja tecnología. El objetivo del presente estudio fue evaluar un indicador con base en estrés térmico para calendarizar el riego diario para el cultivo de tomate (Solanum lycopersicum) cultivado en invernadero. Se estimó un índice de estrés térmico (IET) a partir de las diferencias entre temperatura óptima para la fotosíntesis de tomate y la temperatura del aire y del follaje. Con el IET se estiman los horarios en los que se aplica la lámina de riego calculada a partir de un minievaporímetro tipo B y el coeficiente del cultivo $(\mathrm{Kc})$ de tomate. Los resultados indican que se deben aplicar de cinco a ocho riegos por día con tiempos de riego no mayores a $12 \mathrm{~min}\left(2 \mathrm{a} \mathrm{mm} \mathrm{d}^{-1}\right)$. El volumen aplicado fue de $1.8 \mathrm{~L} /$ planta, similar a lo estimado en estudios con sensores de flujo de savia. Esta metodología permite estimar calendario de riego y simplificar su manejo. El uso del minievaporimetro tipo $\mathrm{B}$, permitió estimar la lámina de riego diaria a partir de la temperatura del aire y cultivo para identificar el estrés térmico y con ello inferir el momento del riego durante el día.

Palabras clave: Solanum lycopersicum, estrés térmico, horario de riego, minievaporimetro tipo $\mathrm{B}$, tomate.

\section{SUMMARY}

The use of sensors and actuators allows the precise application of irrigation through automated and feedback systems; however, its high cost makes it difficult to adopt in medium to low-technology greenhouses. The aim of this study was to evaluate an indicator based on thermal stress to schedule the daily irrigation in tomato (Solanum lycopersicum) grown under a greenhouse. A thermal stress index (TSI) was estimated from the differences between the optimum temperature for tomato photosynthesis and the air and foliage temperature. With the TSI, the schedule in which the irrigation sheet is applied, calculated from a type B mini-evaporimeter and the tomato crop coefficient $(\mathrm{Kc})$ were estimated. Results indicate that five to eight irrigations per day should be applied with irrigation times no longer than $12 \mathrm{~min}(2$ to $4 \mathrm{~mm} \mathrm{~d}^{-1}$ ). The applied volume was $1.8 \mathrm{~L} /$ plant, similar to that estimated in studies employing sap flow sensors. This methodology allows estimating the irrigation calendar and simplifying its management. The use of type B minievaporimeter allowed the estimating of the daily irrigation sheet from the air and crop temperature to identify thermal stress and thereby infer the time of irrigation during the day.

Index words: Solanum lycopersicum, irrigation schedule, thermal stress, tomato, type B mini-evaporimeter

\section{INTRODUCCIÓN}

Con el reto de mantener la producción de cultivos con el menor uso posible de recursos, los sistemas de producción han evolucionado en el cálculo preciso del requerimiento de riego, sobre todo en regiones con escasez de agua (Knox et al., 2012). A ello se suma la agricultura protegida, que además de mantener un ambiente en intervalos aptos para los cultivos, incrementa la productividad del agua. Para definir la lámina de riego existen métodos directos (lisímetro de pesada y de balance) e indirectos (micrometeorológicos y agronómicos), siendo los modelos de Pennman-Monteith, Medrano, Montero, Stanghellini, Fyn y otros los más usados dentro de los indirectos (Li et al., 2016; Ta et al., 2012; Tagliaferre et al., 2013; Tsitsimpelis et al., 2016), mientras que para definir el momento de riego y lámina de riego, las metodologías se pueden clasificar por las variables del suelo, climáticas y del cultivo (CuellarMurcia y Suárez-Salazar, 2018; Medrano et al., 2005; Nikolaou et al., 2019; Shin et al., 2014; Vence et al., 2013); no obstante, éstas tecnologías aún son inaccesibles para productores de baja y mediana tecnología porque implican inversiones costosas.

Rojas et al. (2003) propusieron el método del tiempo térmico acumulado, con el que pudieron calcular 12 riegos en promedio por día; también se ha usado el índice de estrés hídrico del cultivo (Crop Water Stress Index -CWSI), el cual expresa estrés en términos de balance energético y está incluido en el análisis de imágenes para determinar el momento de riego como parte de la agricultura 4.0 (Zhang 
et al., 2019). Smigaj et al (2017) indicaron que es posible detectar el estrés térmico mediante termografía utilizando minicámaras, siendo efectivo para diversos tipos de clima y con el mismo principio del balance térmico. Burke et al. (1990) propusieron un índice de estrés térmico para que reflejara la condición fisiológica del cultivo a lo largo del día, ya que el estrés por calor disminuye la actividad de la enzima Rubisco, la síntesis de clorofila y el transporte de electrones (Hermida-Carrera et al., 2016; Fahad et al., 2017), lo cual puede ocurrir aún con humedad suficiente (Lu et al., 2017; Yokoyama et al., 2019).

Entre los propósitos de cultivar en ambientes protegidos se incluye evitar estrés hídrico que impacte en la disminución del rendimiento (Golam et al., 2012; Yokoyama et al., 2019). El objetivo de este estudio fue proponer y evaluar un indicador con base en el estrés térmico (Índice de estrés térmico -IET) de bajo costo para calendarizar el riego diario de un cultivo de tomate en invernadero.

\section{MATERIALES Y MÉTODOS}

El registro de variables se hizo en un invernadero tipo "diente de sierra" (0.8 ha) con cubierta de polietileno blanco lechoso, calibre 700 y $20 \%$ sombra, ubicado en San Vicente Solís, Temascalcingo, Estado de México. El sistema de riego fue por goteo con gotero insertado de $4 \mathrm{LPH}\left(\mathrm{L} \mathrm{h}^{-1}\right)$ con dos estacas de $2 \mathrm{LPH}$, las cuales fueron colocadas una por planta. El sistema estaba semiautomatizado con un temporalizador industrial y electroválvulas que permitieron adecuar el riego de forma empírica durante el ciclo de 3 a 12 min en función de la etapa de máxima demanda.

El tomate comercial El Cid $F_{1}$ (Harris Moran ${ }^{\circledR}$ ) fue establecido en suelo de textura arcillo arenosa con $0.0914 \mathrm{~m}$ de humedad aprovechable el 18 de junio de 2017; el cultivo se condujo a doble tallo a una densidad de plantación de 2.7 tallos $\mathrm{m}^{-2}$ (40 $\mathrm{cm}$ entre matas y $1.65 \mathrm{~m}$ entre hileras). La temperatura del aire y la humedad relativa se registraron cada día con un termómetro de mercurio de máximas y mínimas de -30 a $50{ }^{\circ} \mathrm{C}$ (Herter ${ }^{\circledR}$, Herter Instruments, Barcelona, España) y un termohigrómetro digital de -10 a $60{ }^{\circ} \mathrm{C}\left(\right.$ TFA $^{\oplus}$, TFA Dostmann, Wertheim-Reicholzheim, Alemania), respectivamente. La temperatura de la planta se midió con un termómetro infrarrojo de pistola portátil con capacidad de medición de -20 a $350^{\circ} \mathrm{C}$, y precisión de $\pm 2.5{ }^{\circ} \mathrm{C}$, tiempo de respuesta $500 \mathrm{~ms}$, longitud de onda 630-670 nm (Modelo Her-425 ${ }^{\circledR}$, San Diego, California, EUA) en el tercio superior de la planta en la hoja recientemente madura y expuesta a la radiación solar, haciendo un recorrido cercano al borde $(1-2 \mathrm{~cm}$ del borde aproximadamente) del foliolo medio de la hoja (Righi et al., 2012). La temperatura del aire y planta se registró una vez por semana, considerando que la inercia de la radiación permanece invariable durante ese periodo y resultaría práctico para el productor. Se midió en 12 plantas al azar en la parte media y orillas, este y oeste del invernadero durante la hora de máxima insolación.

\section{Cálculo de requerimiento de riego (Lámina de riego aplicada - Lra)}

El cálculo de la Lra se estimó asumiendo una eficiencia del riego de 0.90, según Irmak et al. (2011), por lo que la lámina de riego se deriva de la Ec. 1.

$$
L r a=\frac{R R}{E f r}
$$

Donde Lra es la lámina de riego aplicada (mm), Efr es la eficiencia de aplicación del sistema de riego por goteo 0.90 (adimensional) y RR es el requerimiento de riego o evapotranspiración real del cultivo, ETc (mm).

Para estimar la evapotranspiración real del cultivo, se ajustó el modelo usado por Allen et al. (2006) de la Ec. 2.

$$
E T c=(E t o)(F T)(K c)
$$

Ec. 2

Donde ETc es la evapotranspiración real del cultivo $(\mathrm{mm})$, ETo es la evapotranspiración de referencia $\left(\mathrm{mm} \mathrm{d}^{-1}\right)$ método tanque tipo B, FT es el factor de tanque, de tablas con factores climáticos (0.8) y Kc el coeficiente de cultivo (adimensional).

Se construyó un minievaporímetro tipo B con tapón de PVC sanitario clase norma M6, el cual fue colocado en el pasillo central del invernadero sobre una base de madera de $5 \mathrm{~cm}$ de altura para separarlo del suelo, que estaba cubierto con malla blanca (Figura 1). El agua se renovó cada 3 días para marcar el nivel inicial y final cada 24 horas (en mm) para ajustar la lámina de riego aplicada (Lra) diaria. El FT elegido fue de 0.8, correspondiente al caso B, caracterizado por viento ligero y humedad relativa media (Romero et al., 2009).

El Kc que se usó fue el valor máximo reportado por ValdésGómez et al. (2009), que corresponde a un invernadero con ventilación natural; este coeficiente se ajusta diariamente en función de la altura del cultivo y la humedad relativa mínima con la Ec. 3 (Čereković et al., 2010). El dato de la velocidad del viento se tomó de la bibliografía como un promedio máximo medido, reportado para invernaderos con ventilación natural, con valor promedio de $1.38 \mathrm{~m} \mathrm{~s}^{-1}$ (5 $\mathrm{km} \mathrm{h}^{-1}$ ) (Kumar et al., 2009; Villagrán et al., 2012) debido a que no puede controlarse la velocidad del viento a $1 \mathrm{~m} \mathrm{~s}^{-1}$ como lo indica la ASABE (2003). 


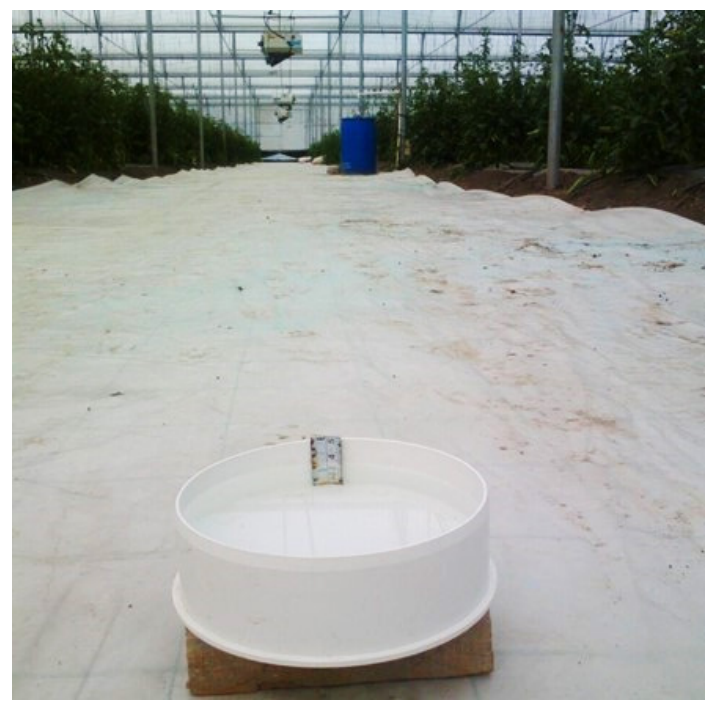

Figura 1. Ubicación del minievaporimetro tipo B (tapón de PVC sanitario clase norma M6) en el invernadero.

$$
K C_{\text {mid }}=K C_{\text {mid(Tab) }}+\left[\left(0.04 U_{2}-2\right)-0.004\left(H R_{\text {min }}-45\right)\right]\left(\frac{h}{3}\right)^{0.3}
$$

donde $\mathrm{Kc}_{\text {mid }}$ es el coeficiente del cultivo promedio, $\mathrm{Kc}_{\text {mid }}$ (Tab) es 1.06 y 1.25 para el periodo muestreado, $U_{2}$ es la velocidad del viento tabulada a $2 \mathrm{~m}$ de altura $\left(1.38 \mathrm{~m} \mathrm{~s}^{-1}\right)$, $\mathrm{HR}_{\text {min }}$ es la humedad relativa mínima diaria (\%) y h es la altura del cultivo $(\mathrm{m})$.

El Kc de la Ec. 2 se sustituyó por $\mathrm{Kc}_{\text {mid }}$ (Ec.3) para calcular la evapotranspiración del tomate. Una vez obtenida la Lra en $\mathrm{mm}$, ésta se expresó en $\mathrm{mL} \mathrm{m}^{-2}$ usando $1 \mathrm{~m}^{2}$ de superficie de suelo $\left(1,000,000 \mathrm{~mm}^{2}\right)$ para calcular el volumen de riego por planta o tallo $(\mathrm{Vr})$ como se indica en la Ec. 4. Con dichos valores y el caudal promedio $(\mathrm{mL}$ $\mathrm{min}^{-1}$ ) de los goteros medido en invernadero se calculó el tiempo de riego $(\mathrm{Tr})$ en min, considerando dos goteros/ planta (Ec. 5).

$$
\mathrm{Vr}=\frac{\mathrm{Lra}}{\mathrm{Dt}}
$$

Ec. 4

Donde $V r$ es el volumen de riego $(\mathrm{mL})$, Lra es la lámina de riego aplicada $\left(\mathrm{mL} \mathrm{m}^{-2}\right)$, Dt es la densidad de plantas (2.7 plantas $\left.\mathrm{m}^{-2}\right)$.

$$
\operatorname{Tr}=\frac{\mathrm{Vr}}{\mathrm{Qg}}
$$

Donde $\operatorname{Tr}$ es el tiempo de riego (min), $\mathrm{Vr}$ es el volumen de riego $(\mathrm{mL})$, Qg es el gasto del gotero $\left(\mathrm{mL} \mathrm{min}{ }^{-1}, 32 \times 2\right.$ goteros en este caso).

\section{Índice de estrés térmico}

El índice de estrés térmico horario (IETh) se calculó usando la Ec. 6. Se consideró el intervalo de temperatura de 18 a $25.5^{\circ} \mathrm{C}$ como el óptimo para la fotosíntesis del cultivo de tomate (Shamshiri et al., 2018); con ello, se calcularon las relaciones Ta-25.5, Th-25.5, 18-Ta y 18-Th y se tomaron en cuenta sus valores positivos.

$$
\mathrm{IET}_{\mathrm{h}}=\frac{\frac{(\mathrm{Ta}-25.5)+(18-\mathrm{Ta})}{2}+\frac{(\mathrm{Th}-25.5)+(18-\mathrm{Th})}{2}}{2}
$$

donde: IET ${ }_{h}$ es el índice de estrés térmico horario $\left({ }^{\circ} \mathrm{C}\right)$, Ta es la temperatura del aire horaria $\left({ }^{\circ} \mathrm{C}\right)$, Th es la temperatura de la hoja horaria medida con termómetro infrarrojo $\left({ }^{\circ} \mathrm{C}\right)$.

Sumando los valores del IET se obtuvo el IET global $\left(\mathrm{IET} \mathrm{q}_{\mathrm{g}}\right.$; posteriormente, se definió en índice de estrés térmico estandarizado ( $\mathrm{ET}_{\text {h0-1 }}$ ), el cual se obtuvo dividiendo $\mathrm{IET}_{\mathrm{h}}$ entre IET $\mathrm{h}_{\mathrm{h}-1}$ para obtener valores en una escala de 0-1 (Cuadro 1); con ello fue posible calcular el horario y tiempo de riego. El tiempo de riego se afina atendiendo el tiempo mínimo de riego y el estrés térmico detectado para reducir los eventos de riego, ya que se estima un incremento en el uso del sistema de bombeo (Flórez-Tuta et al., 2013). Para los datos del 8 de agosto de 2017 (Cuadro 1) se calculó un volumen de riego en $1.78 \mathrm{~L}$ por planta, equivalentes a un tiempo de riego de $27.81 \mathrm{~min}$. Con estos datos se estima el tiempo de riego horario (Tr), del que se obtiene el tiempo de riego ajustado $\left(\mathrm{Tr}_{\mathrm{a}}\right)$ en min, eliminando las fracciones (redondeo) y acumulando éstas al siguiente riego, como se muestra en Cuadro 1.

Con esta metodología es factible estimar diariamente los momentos de riego en función de la variación del Kc y, en general, de las variables involucradas en el calculó de la ETo diaria. El horario del Cuadro 1 se estableció para el riego de toda la semana, detectándose variaciones únicamente en el volumen de riego en días nublados (Figura 3B), donde no había estrés y el riego de ese horario se suspendió.

\section{RESULTADOS Y DISCUSIÓN}

Al analizar la termometría de la planta pudo observarse que la temperatura del fruto fue mayor que la del ambiente (Figura 2A), por lo cual fue descartado como órgano de referencia. No obstante, la temperatura de la hoja (Th) y del aire ( $\mathrm{Ta}$ ) se incrementan siguiendo el mismo patrón; sin embargo, la Th alcanza un máximo a pesar de que la Ta continúa aumentando, registrándose gradientes térmicos de 3 a $7{ }^{\circ} \mathrm{C}$ (Figura 2B). A partir de ese momento, la planta utiliza mecanismos para mantener su temperatura (Buckley, 2005), ocurriendo el estrés térmico como se ha indicado en algodón (Burke et al., 1990). El registro 
Cuadro 1. Horario y tiempo de riego calculados a partir del tiempo de riego diario.

\begin{tabular}{|c|c|c|c|c|c|c|}
\hline \multirow{2}{*}{ Hora del día } & $\mathrm{Ta}$ & Th & $\mathrm{IET}_{\mathrm{h}}$ & \multirow{2}{*}{$\mid E T_{\text {ho-1 }}$} & $\operatorname{Tr}$ & $\mathrm{Tr}_{\mathrm{a}}$ \\
\hline & & ${ }^{\circ} \mathrm{C}$ & & & \multicolumn{2}{|c|}{$\min$} \\
\hline 8:00 & 11.00 & 9.74 & 3.82 & 0.12 & 3.34 & 3 \\
\hline 9:00 & 18.00 & 16.34 & 0.42 & 0.01 & 0.28 & \\
\hline 10:00 & 23.00 & 18.36 & 0.00 & 0.00 & 0.00 & \\
\hline 11:00 & 27.00 & 23.63 & 1.72 & 0.05 & 1.39 & \\
\hline 12:00 & 30.50 & 25.83 & 4.89 & 0.16 & 4.45 & 6 \\
\hline 13:00 & 32.50 & 27.01 & 6.68 & 0.21 & 5.84 & 6 \\
\hline 14:00 & 30.00 & 23.68 & 3.40 & 0.11 & 3.06 & 3 \\
\hline 15:00 & 32.00 & 25.24 & 5.72 & 0.18 & 5.00 & 5 \\
\hline 16:00 & 29.00 & 22.43 & 3.09 & 0.10 & 2.78 & 4 \\
\hline \multirow[t]{2}{*}{ 17:00 } & 26.00 & 23.43 & 1.69 & 0.05 & 1.39 & \\
\hline & \multicolumn{2}{|c|}{$\Sigma \mid \mathrm{ET}_{\mathrm{h+}}=\mathrm{IET}$} & 31.43 & 1.00 & 27.53 & 27 \\
\hline
\end{tabular}

Ta: temperatura del aire, Th: temperatura de la hoja, IETh : índice de estrés térmico horario $\left({ }^{\circ} \mathrm{C}\right)$, IET $\mathrm{EL}_{\mathrm{h}-1}$ : índice de estrés térmico estandarizado a 0-1, Tr: tiempo de riego, $\operatorname{Tr}_{a}$ : tiempo de riego ajustado.

fenológico permitió definir periodos de crecimiento del cultivo y el Kc, siendo el inicio de la floración, inicio de fructificación e inició de cosecha los más importantes. El número de hojas se incrementó de forma exponencial hasta 22 aproximadamente, realizando la primera poda a los 59 días después del trasplante (DDT), y de manera semanal a partir de esta fecha que se registró como los altibajos que se observan en la Figura 2C.

La evaporación registrada con el minievaporímetro tipo B mostró valores máximos de $6 \mathrm{~mm} \mathrm{~d}^{-1}$, siendo frecuentes intervalos de ETc de 2 a $4 \mathrm{~mm} \mathrm{~d}{ }^{-1}$. Estos valores son similares a los reportados por Čereković et al. (2010) y Ortega-Farias et al. (2000) en condiciones de invernadero, que corresponde a requerimientos hídricos de 0.9 a 1.82 $\mathrm{L} /$ planta. Valores similares fueron también indicados por Flores et al. (2007), quienes usaron sensores de flujo de savia, lisímetro de balance y Penman-Monteith modificado por FAO para tomate en invernadero. Otros investigadores han reportados valores similares en invernadero usando atmómetro, minievaporímetro UFV-1, modelo PenmannMonteith y el tanque evaporímetro Tipo A (Blanco y Folegatti, 2004; Giambelluca et al., 1992; Ondrašek et al., 2007; Romero et al., 2009; Tagliaferre et al., 2013; Worth et al., 1994). La propuesta de este trabajo es un método de aplicación práctica para elegir el momento de aplicación del riego.

En el IET $\mathrm{h}_{\mathrm{h}}$ de la Figura 3 se observa que el mayor estrés por temperaturas altas se presenta desde las 12:00 hasta las 16:00-17:00 horas. Investigaciones similares señalan que durante periodos de estrés la fisiología del tomate tiene mecanismos para tomar agua de sus frutos para la evapotranspiración, en casos extremos, afectando de manera negativa la calidad de los mismos (De Swaef et al., 2012). En un día con periodos nublados (Figura 3B) el estrés de la planta disminuye, como se observa en el horario de las 14:00 horas, pues se ha estimado que el tiempo de respuesta fisiológica del tomate a la falta de agua es de aproximadamente 5 min (Lee, 2013). En octubre el IET disminuye (Figura 3D); sin embargo, el cultivo está sometido a estrés por temperaturas bajas y altas afectando la maduración y calidad de los frutos, como ya se ha indicado en otras investigaciones (Liu et al., 2013; Kacjan Maršić et al., 2011; Lizarraga et al., 2003;).

Con el IET se determinaron de tres a siete eventos de riego, aunque se aplicaron hasta ocho; un número menor a los estimados por Rojas et al. (2003), lo cual significó un ahorro en el consumo de energía en comparación con los sistemas retroalimentados (Nikolaou et al., 2019). En tomate cultivado en tezontle y suelo, Ojodeagua et al. (2008) aplicaron de 8 a 15 riegos de 3 min por día y concluyeron que en suelo hay un ahorro de agua de 24 a $30 \%$. El 11 de septiembre a las 08:00 a.m. se determinó un tiempo de riego mayor a 12 min, para evitar sobre-riego se decidió fraccionarlo en dos aplicaciones, la mitad en el horario indicado y el resto con el siguiente horario de media hora (08:30 a.m.). Bajo las condiciones expuestas, se estimó la relación entre los $\mathrm{mL}$ de agua por grado centígrado que es necesario aplicar en tiempo real, la cual varía a lo largo del ciclo de cultivo, como se muestra en el Cuadro 3.

El rendimiento del cultivo se estimó en aproximadamente 

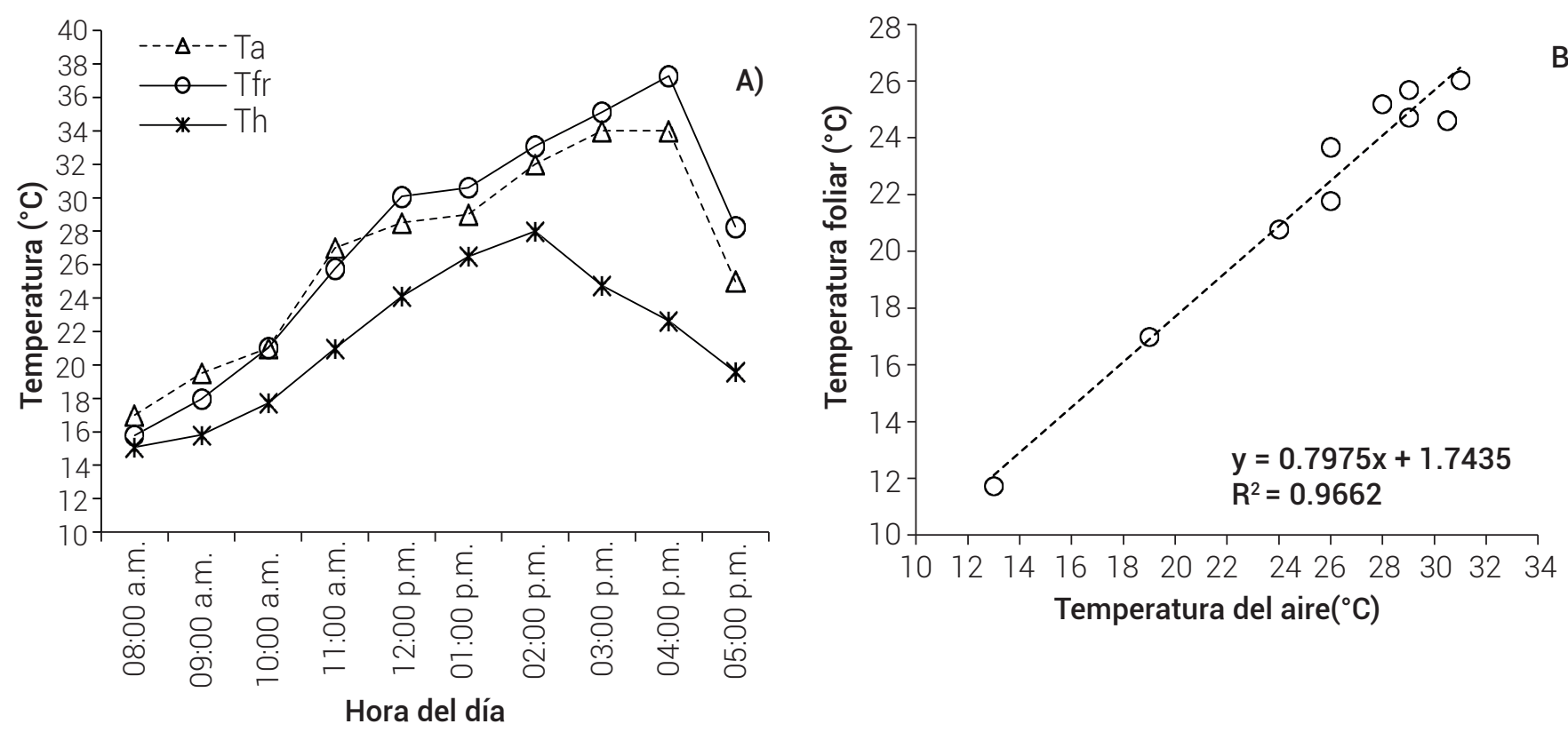

B)

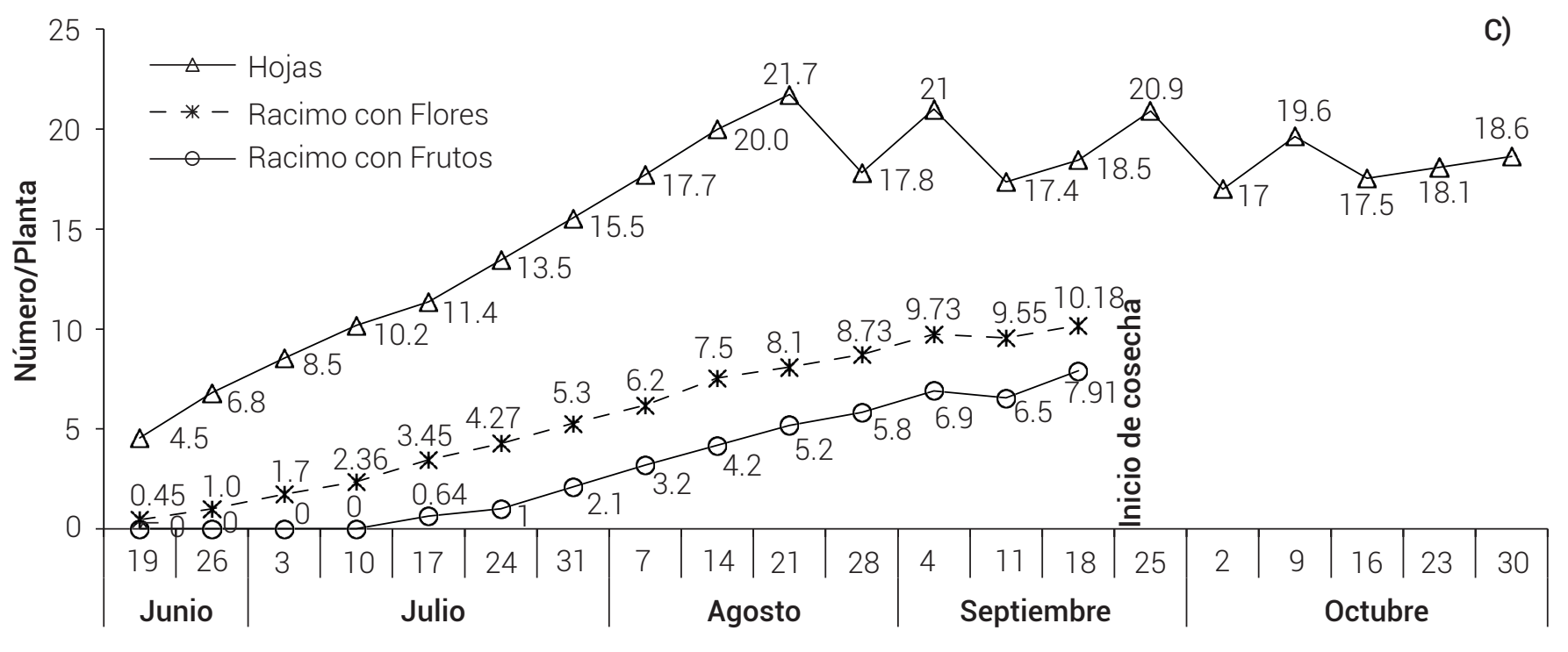

Fecha después del trasplante

Figura 2. A) Temperatura del aire, de frutos y hojas de tomate $(n=12), B)$ relación entre la temperatura del aire y de la hoja $(n=12)$ y $C)$ fenología del jitomate en invernadero. Ta: temperatura del aire, Tfr. temperatura del fruto, Th: temperatura de la hoja.

Cuadro 2. Estimación del momento y duración del riego en tomate en invernadero.

\begin{tabular}{lccccccccccc}
\hline Variables & \multicolumn{10}{c}{ Semanas de crecimiento } \\
& 5 & 6 & 7 & 8 & 9 & 10 & 11 & 12 & 13 & 14 & 15 \\
\hline AP (cm) & 60.70 & 87.80 & 107.90 & 139.70 & 161.20 & 179.30 & 203.40 & 236.10 & 241.50 & 259.90 & 283.6 \\
Kc & 1.06 & 1.25 & 1.25 & 1.25 & 1.25 & 1.25 & 1.25 & 1.25 & 1.25 & 1.25 & 1.25 \\
ETc & 2.30 & 5.37 & 2.70 & 4.35 & 2.20 & 2.20 & 2.21 & 4.41 & 5.51 & 3.33 & 2.79 \\
HRm (\%) & 20.00 & 25.00 & 25.00 & 38.00 & 40.00 & 50.00 & 44.00 & 51.00 & 48.00 & 50.00 & 39.00 \\
\hline
\end{tabular}

AP. altura de planta, ETc: evapotranspiración del cultivo, HRm: humedad relativa mínima. 


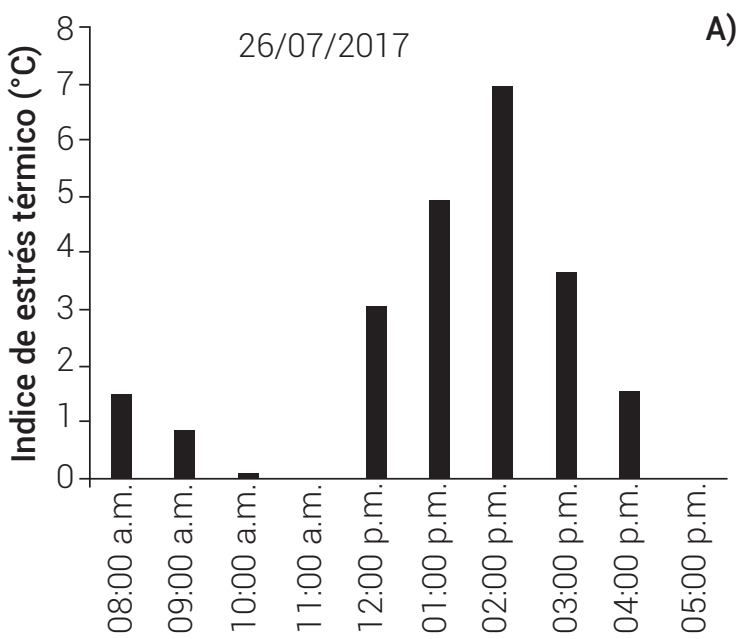

Hora del día

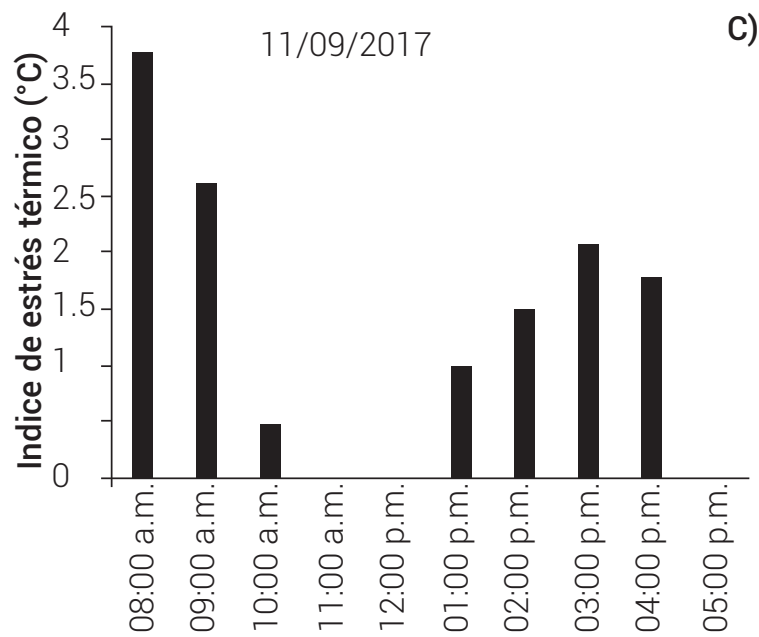

Hora del día

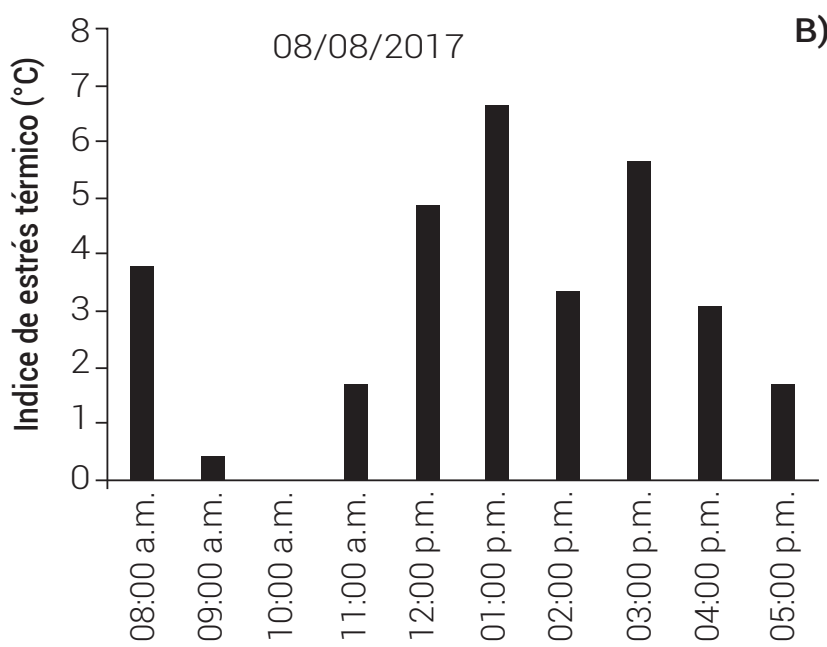

Hora del día

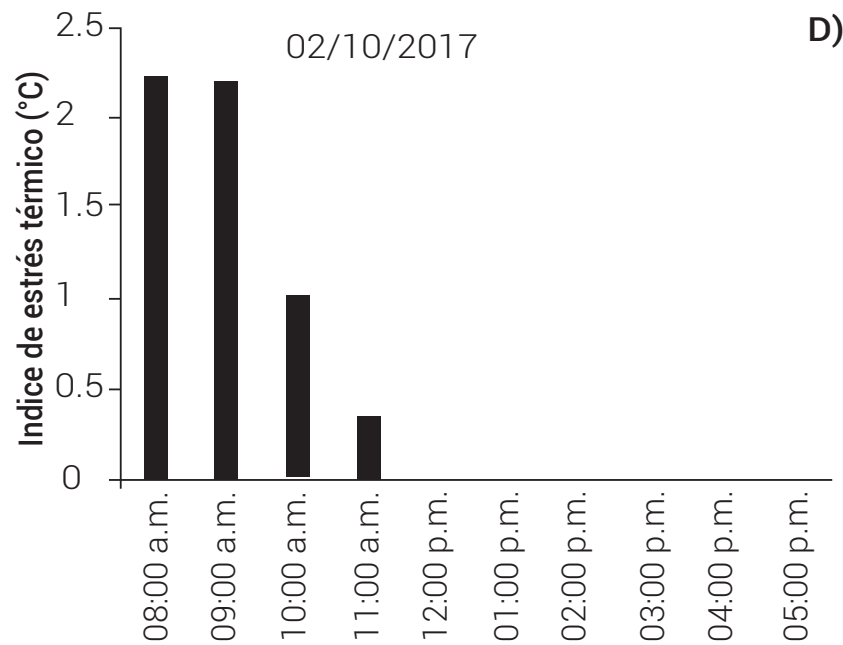

Hora del día

Figura 3. Índice de estrés térmico horario (IET $)$ del tomate en invernadero en cuatro días soleados: A) julio, B) agosto, C) septiembre y D) octubre.

$165 \mathrm{t} \mathrm{ha}^{-1}$ (valor extrapolado), cuya calidad de frutos fue principalmente extra y primera (58.88\%), con aproximadamente $13.94 \%$ de "canica" debido a la falta de polinización; mientras tanto, en la categoría de segunda y tercera se obtuvo $20.82 \%$ y $6.34 \%$, respectivamente. La merma se debió principalmente a frutos con cicatriz carpelar y deformaciones del fruto. De acuerdo con datos del SIAP (2020), el rendimiento promedio nacional fue de $168.48 \mathrm{t} \mathrm{ha}^{-1}$, apenas 3.5 toneladas más que el registrado en el ciclo evaluado con el hibrido El Cid $\mathrm{F}_{1}$.

\section{CONCLUSIONES}

Se contribuyó en el proceso de calendarización del riego en invernaderos de mediana-baja tecnología mediante el uso de un tanque evaporímetro Tipo B para estimar una lámina de riego diaria y mediciones de temperatura para definir el momento de la aplicación del riego. Con esta propuesta metodológica fue posible estimar volúmenes de riego, bajo las condiciones regionales de Temascalcingo, Estado de México, en el intervalo de 0.95 a $1.82 \mathrm{~L} /$ planta/ día durante el ciclo. Mediante el Índice de Estrés Térmico horario ( $\mathrm{IET}_{h}$ ) propuesto fue posible proponer el horario y duración del riego. Utilizando el IET ${ }_{h}$ se determinaron entre cinco y ocho eventos de riego diarios con duración de entre tres y ocho minutos.

\section{BIBLIOGRAFÍA}

Allen R. G., S. Pereira L., D. Raes y M. Smith (2006) Evapotranspiración del Cultivo. Guías para la Determinación de los Requerimientos de Agua de los Cultivos. Organización de las Naciones Unidas para la Alimentación y la Agricultura. Roma, Italia. 300 p.

ASABE, American Society of Agricultural and Biological Engineers (2003) Heating, ventilating and cooling greenhouses. ANSI/ASAE Standard EP406.4. American Society of Agricultural and 
Cuadro 3. Eventos y tiempos de riego calculados en un día soleado de cuatro semanas.

\begin{tabular}{|c|c|c|c|c|c|c|c|c|c|c|c|c|}
\hline & \multicolumn{3}{|c|}{$26 / 07 / 2017$} & \multicolumn{3}{|c|}{ 08/08/2017 } & \multicolumn{3}{|c|}{ 11/09/2017 } & \multicolumn{3}{|c|}{$02 / 10 / 2017$} \\
\hline $\operatorname{Vr}(\mathrm{mL} /$ planta $)$ & \multicolumn{3}{|c|}{950} & \multicolumn{3}{|c|}{1780} & \multicolumn{3}{|c|}{1820} & \multicolumn{3}{|c|}{1150} \\
\hline $\operatorname{Tr}(\min )$ & \multicolumn{3}{|c|}{14.8} & \multicolumn{3}{|c|}{27.8} & \multicolumn{3}{|c|}{28.4} & \multicolumn{3}{|c|}{17.9} \\
\hline Horario & $\mathrm{IET}_{\text {h0-1 }}$ & $\mathrm{Tr}_{0-1}$ & $\operatorname{Tr}_{\mathrm{a}}$ & $\mathrm{IET}_{\text {h0-1 }}$ & $\operatorname{Tr}_{0-1}$ & $\operatorname{Tr}_{\mathrm{a}}$ & $I E T_{\text {h0-1 }}$ & $\operatorname{Tr}_{0-1}$ & $\mathrm{Tr}_{\mathrm{a}}$ & $\mid E T_{\text {h0-1 }}$ & $\operatorname{Tr}_{0-1}$ & $\operatorname{Tr}_{\mathrm{a}}$ \\
\hline 08:00 & 0.066 & 0.97 & & 0.121 & 3.36 & 3 & 0.286 & 8.12 & 8 & 0.385 & 6.89 & 6 \\
\hline \multicolumn{13}{|l|}{ 08:30 } \\
\hline 09:00 & 0.036 & 0.53 & & 0.013 & 0.36 & & 0.199 & 5.56 & 5 & 0.381 & 6.82 & 7 \\
\hline 10:00 & 0.003 & 0.04 & & 0.000 & 0.00 & & 0.036 & 1.02 & & 0.175 & 3.13 & 4 \\
\hline 11:00 & 0.000 & 0.00 & & 0.055 & 1.53 & & 0.000 & 0.00 & & 0.059 & 1.05 & \\
\hline 12:00 & 0.134 & 1.98 & 3 & 0.156 & 1.33 & 3 & 0.000 & 0.00 & & 0.000 & 0.00 & \\
\hline 13:00 & 0.221 & 3.27 & 4 & 0.213 & 5.92 & 6 & 0.076 & 2.16 & 4 & 0.000 & 0.00 & \\
\hline 14:00 & 0.309 & 4.57 & 4 & 0.108 & 3.00 & 3 & 0.113 & 3.21 & 3 & 0.000 & 0.00 & \\
\hline 15:00 & 0.163 & 2.41 & & 0.182 & 5.06 & 5 & 0.157 & 4.46 & 4 & 0.000 & 0.00 & \\
\hline 16:00 & 0.067 & 0.99 & 4 & 0.098 & 2.72 & 3 & 0.133 & 3.78 & 4 & 0.000 & 0.00 & \\
\hline 17:00 & 0.000 & 0.00 & & 0.054 & 1.50 & 3 & 0.000 & 0.00 & & 0.000 & 0.00 & \\
\hline $\mathrm{IET}_{\mathrm{g}}\left({ }^{\circ} \mathrm{C}\right)$ & & 22.38 & & & 31.43 & & & 13.23 & & & 5.78 & \\
\hline $\mathrm{mL}^{\circ}{ }^{\circ} \mathrm{C}^{-1}$ & & 42.45 & & & 56.63 & & & 137.56 & & & 198.96 & \\
\hline
\end{tabular}

Vr: volumen de riego, $\mathrm{Tr}$ : tiempo de riego estimado, IET : índice de estrés térmico en escala 0-1, $\mathrm{Tr}_{0-1}$ : tiempo de riego estandarizado en escala 0-1, $\mathrm{Tr}_{a}$ : tiempo de riego ajustado sin fracciones y tiempo de riego $\geq 3 \mathrm{~min} \mathrm{y} \leq 12 \mathrm{~min}$.

Biological Engineers. St. Joseph, Michigan. 9 p.

Blanco F. F. and M. V. Folegatti (2004) Evaluation of evaporationmeasuring equipments for estimating evapotranspiration within a greenhouse. Revista Brasileira de Engenharia Agricola e Ambiental 8:184-188, https://doi.org/10.1590/S141543662004000200004

Buckley T. N. (2005) The control of stomata by water balance. New Phytologist 168:275-292, https://doi.org/10.1111/j.14698137.2005.01543.x

Burke J. J., J. L. Hatfield and D. F. Wanjura (1990) A thermal stress index for cotton. Agronomy Journal 82:526-530, https://doi. org/10.2134/agronj1990.00021962008200030018x

Čereković N., M. Todorović, R. L. Snyder, F. Boari, B. Pace and V. Cantore (2010) Evaluation of the crop coefficients for tomato crop grow in a Mediterranean climate. In: Economics of Drought and Drought Preparedness in a Climate Change Context. A. López-Francos (comp.). Centre International de Hautes Etudes Agronomiques Méditerranéennes. Paris, France. pp:91-94.

Cuellar-Murcia C. A. y J. C. Suárez-Salazar (2018) Flujo de savia y potencial hídrico en plantas de tomate (Solanum lycopersicum L.) bajo condiciones de invernadero. Revista Colombiana de Ciencias Hortícolas 12:104-112, https://doi.org/10.17584/ rcch.2018v12i1.7316

De Swaef T., K. Verbist, W. Cornelis and K. Steppe (2012) Tomato sap flow, stem and fruit growth in relation to water availability in rockwool growing medium. Plant and Soil 350:237-252, https:// doi.org/10.1007/s11104-011-0898-4

Fahad S., A. Bajwa A., U. Nazir, S. A. Anjum, A. Farooq, A. Zohaib, ... and J. Huang (2017) Crop production under drought and heat stress: plant responses and management options. Frontiers in Plant Science 8:1147, https://doi.org/10.3389/fpls.2017.01147

Flores J., W. Ojeda-Bustamante, I. López, A. Rojano e I. Salazar (2007) Requerimientos de riego para tomate de invernadero. Terra Latinoamericana 25:127-134

Flórez-Tuta N., I. Zution-Gonçalves, D. Rodrigues-Calvacante Feitosa, E. A. Agnellos-Barbosa, F. Ponciano-de Deus, M. Diego-Ribeiro y E. EijiMatsura (2013) Eficiencia de aplicación de agua en la superficie y en el perfil del suelo en un sistema de riego por aspersión.
Agrociencia 47:107-119.

Giambelluca T. W., L. Mckenna D. and P. C. Ekern (1992) An automated recording atmometer: I. calibration and testing. Agricultural and Forest Meteorology 62:109-125, https://doi.org/10.1016/01681923(92)90008-R

Golam F., Z. H. Prodham, A. Nezhadahmadi and M. Rahman (2012) Heat tolerance in tomato. Life Science Journal 9:1936-1950.

Hermida-Carrera C., M. V. Kapralov and J. Galmés (2016) Rubisco catalytic properties and temperature response in crops. Plant Physiology 171:2549-2561, https://doi.org/10.1104/pp.16.01846

Irmak S., O. Odhiambo L., L. Kranz W., D. E. Eisenhauer (2011) Irrigation efficiency and uniformity, and crop water use efficiency. Papers and Publications 451. Biological Systems Engineering. University of Nebraska. Lincoln, Nebraska, USA. 8 p.

Kacjan Maršić N., L. Gašperlin, V. Abram, M. Budič and R. Vidrih (2011) Quality parameters and total phenolic content in tomato fruits regarding cultivar and microclimatic conditions. Turkish Journal of Agriculture and Forestry 35:185-194, https://doi org/10.3906/tar-0910-499

Knox J. W., M. G. Kay and E. K. Weatherhead (2012) Water regulation, crop production, and agricultural water managementUnderstanding farmer perspectives on irrigation efficiency Agricultural Water Management 108:3-8, https://doi. org/10.1016/j.agwat.2011.06.007

Kumar K. S., N. Tiwari K. and M. K. Jha (2009) Design and technology for greenhouse cooling in tropical and subtropical regions: a review. Energy and Buildings 41:1269-1275, https://doi.org/10.1016/j. enbuild.2009.08.003

Lee J. S. (2013) Do really close stomata by soil drying ABA produced in the roots and transported in transpiration stream? American Journal of Plant Sciences 4:169-173, https://doi.org/10.4236/ ajps.2013.41022

Li Y., C. Liu, K and Liang (2016) Spatial patterns and influence factors of conversion coefficients between two typical pan evaporimeters in China. Water 8:422, https://doi.org/10.3390/w8100422

Liu H., A. Duan, F. Li, J. Sun, Y. Wang and C. Sun (2013) Drip irrigation scheduling for tomato grown in solar greenhouse based on pan evaporation in North China Plain. Journal of Integrative 
Agriculture 12:520-531, https://doi.org/10.1016/S20953119(13)60253-1

Lizarraga A., H. Boerveld, F. Huibers and C. Robles (2003) Evaluating irrigation scheduling of hydroponic tomato in Navarra, Spain. Irrigation and Drainage 52:177-188, https://doi.org/10.1002/ ird.86

Lu T., Z. Meng, G. Zhang, M. Qi, Z. Sun, Y. Liu and T. Li (2017) Sub-high temperature and high light intensity induced irreversible inhibition on photosynthesis system of tomato plant (Solanum lycopersicum L.). Frontiers in Plant Science 8:365, https://doi. org/10.3389/fpls.2017.00365

Medrano E., P. Lorenzo, M. C. Sánchez-Guerrero and J. I. Montero (2005) Evaluation and modelling of greenhouse cucumber-crop transpiration under high and low radiation conditions. Scientia Horticulturae 105:163-175, https://doi.org/10.1016/j. scienta.2005.01.024

Nikolaou G., D. Neocleous, N. Katsoulas and C. Kittas (2019) Irrigation of greenhouse crops. Horticulturae 5:7, https://doi.org/10.3390/ horticulturae 5010007

Ojodeagua A. J. L., J. Z. Castellanos R., J. J. Muñoz R., G. G. Alcántar, L. Tijerina C., P. Vargas T. y S. Enríquez R. (2008) Eficiencia de suelos y tezontle en sistemas de producción de tomate en invernadero. Revista Fitotecnia Mexicana 31:367-374

Ondrašek G., D. Romić, J. Borošić, M. Romić and I. Stričević (2007) Comparison of transpiration models in tomato soilless culture. Agriculturae Conspectus Scientificus 72:113-116.

Ortega-Farias S. O., R. Calderon, C. Acevedo y S. Fuentes (2000) Estimación de la evapotranpiración real diaria de un cultivo de tomates usando la ecuación de Penman-Monteith. Revista Ciencia e Investigación Agraria 27:91-96.

Righi E. Z., G. A. Buriol, L. A. Angelocci, A. B. Heldwein and I. F. Tazzo (2012) Relatioships of photosynthetic photon flux density, air temperature and humidity with tomato leaf diffusive conductance and temperature. Brazilian Archives of Biology and Technology 55:359-370, https://doi.org/10.1590/S151689132012000300005

Rojas A., A. Noriega, G. Herrera y R. Chaparro (2003) Sistema de riego para invernaderos hidropónicos basado en la evapotranspiración del cultivo. Naturaleza y Desarrollo 1:23-29.

Romero E., A. Rodríguez, L. Rázuri, J. Suniaga y E. Montilla (2009) Estimación de las necesidades hídricas del cultivo de pepino (Cucumis sativus L.), durante las diferentes etapas fenológicas, mediante la tina de evaporación. Agricultura Andina 16:56-69.

Shamshiri R. R., J. W. Jones, K. R. Thorp, D. Ahmad, H. C. Man and S. Taheri (2018) Review of optimum temperature, humidity, and vapour pressure deficit for microclimate evaluation and control in greenhouse cultivation of tomato: a review. International Agrophysics 32:287-302, https://doi.org/10.1515/ intag-2017-0005
Shin J. H., J. S. Park and J. E. Son (2014) Estimating the actual transpiration rate with compensated levels of accumulated radiation for the efficient irrigation of soilless cultures of paprika plants. Agricultural Water Management 135:9-18, https://doi. org/10.1016/j.agwat.2013.12.009

SIAP, Servicio de Información Agroalimentaria y Pesquera (2020) Anuario estadístico de la producción agrícola. Servicio de Información Agroalimentaria y Pesquera. Ciudad de México. https://nube. siap.gob.mx/cierreagricola/ (Abril 2021).

Smigaj M., R. Gaulton, J. C. Suarez and S. L. Barr (2017) Use of miniature thermal cameras for detection of physiological stress in conifers. Remote Sensing 9:957, https://doi.org/10.3390/ rs9090957

Ta T. H., J. H. Shin, E. H. Noh and J. E. Son (2012) Transpiration growth, and water use efficiency of paprika plants (Capsicum annum L.) as affected by irrigation frequency. Horticulture, Environment and Biotechnology 53:129-134, https://doi.org/10.1007/s13580012-0095-2

Tagliaferre C., R. A. de Oliveira, G. C. Sediyama, P. R. Cecon, M. A. Martinez and F. J. V. Materán (2013) Performance of the minievaporimeter UFV-1 to estimate the reference evapotranspiration in relation to the constant groundwater table lysimeter. Idesia (Arica) 31:87-99, https://doi.org/10.4067/S0718-34292013000100011

Tsitsimpelis I., I. Walfenden and C. J. Taylor (2016) Development of a grow-cell test facility for research into sustainable controlledenvironment agriculture. Biosystems Engeineering 150:40-53, https://doi.org/10.1016/j.biosystemseng.2016.07.008

Valdés-Gómez H., S. Ortega-Farías and M. Argote (2009) Evaluation of water requirements for a greenhouse tomato crop using the Priestley-Taylor method. Chilean Journal of Agricultural Research 69:3-11.

Vence L. B., O. R. Valenzuela, H. A. Svartiz y M. E. Conti (2013) Elección de sustrato y manejo del riego utilizando como herramienta las curvas de retención de agua. Ciencia del Suelo 31:153-164.

Villagrán E. A., Gil R., J. F. Acuña and C. R. Bojacá (2012) Optimization of ventilation and its effect on the microclimate of a Colombian multispan greenhouse. Agronomía Colombiana 30:282-288.

Worth G. D., F. Holawe and G. N. Mclntyre (1994) An inexpensive and reliable atmometer for estimating evaporation from sandy surfaces. Theoretical and Applied Climatology 49:263-265, https://doi. org/10.1007/BF00867465

Yokoyama G., D. Yasutake, T. Tanizak and M. Kitano (2019) Leaf wetting mitigates depression of photosynthesis in tomato plants. Photosynthetica 57:740-747, https://doi.org/10.32615/ ps.2019.088

Zhang L., H. Zhang, Y. Niu and W. Han (2019) Mapping maize water stress based on UAV multispectral remote sensing. Remote Sensing 11:605, https://doi.org/10.3390/rs11060605 